\title{
TWISTED TRACES OF INTERTWINERS FOR KAC-MOODY ALGEBRAS AND CLASSICAL DYNAMICAL R-MATRICES CORRESPONDING TO GENERALIZED BELAVIN-DRINFELD TRIPLES
}

\author{
Pavel Etingof and Olivier Schiffmann
}

\section{Introduction}

In the early eighties, Belavin and Drinfeld [BD] classified nonskewsymmetric classical r-matrices for simple Lie algebras. It turned out that such r-matrices, up to isomorphism and twisting by elements from the exterior square of the Cartan subalgebra, are classified by rather unusual combinatorial objects which are now called Belavin-Drinfeld triples. By definition, a Belavin-Drinfeld triple for a simple Lie algebra $\mathfrak{g}$ is a triple $\left(\Gamma_{1}, \Gamma_{2}, T\right)$, where $\Gamma_{1}, \Gamma_{2}$ are subsets of the Dynkin diagram $\Gamma$ of $\mathfrak{g}$, and $T: \Gamma_{1} \rightarrow \Gamma_{2}$ is an isomorphism preserving the inner product, which satisfies the nilpotency condition: if $\alpha \in \Gamma_{1}$ then there exists $k$ such that $T^{k-1}(\alpha) \in \Gamma_{1}$ but $T^{k}(\alpha) \notin \Gamma_{1}$. The r-matrix corresponding to such a triple is given by a certain explicit formula. This formula works not only for simple finite dimensional Lie algebras but in fact for any symmetrizable Kac-Moody algebra.

In [S], the second author generalized the work of Belavin and Drinfeld and classified classical nonskewsymmetric dynamical r-matrices for simple Lie algebras. It turns out that they have an even simpler classification: up to gauge transformations, they are classified by generalized Belavin Drinfeld triples, which are defined as the usual Belavin-Drinfeld triples but without any nilpotency condition. The dynamical r-matrix corresponding to such a triple is given by a certain explicit formula, which, as before, works not only for simple finite dimensional Lie algebras but in fact for any symmetrizable Kac-Moody algebra.

This includes some well known examples: if $T=i d$ one gets Felder's dynamical r-matrix, and if $\mathfrak{g}$ is of type $\hat{A}_{n-1}$ (i.e., the Dynkin diagram is an n-gon) and $T$ is the rotation of the n-gon by an angle $2 \pi k / n$ where $k$ is prime to $n$, then one gets Belavin's elliptic r-matrix.

G. Felder $[\mathrm{F}]$ associated to every classical dynamical r-matrix, a remarkable system of differential equations called the Knizhnik-Zamolodchikov-Bernard (KZB) equations. For the Felder and the Belavin r-matrix these equations have a representation-theoretical interpretation. Namely, the KZB equations with the Felder r-matrix are satisfied by conformal blocks for the Wess-Zumino-Witten

Received August 20, 1999. 
(WZW) model of conformal field theory on elliptic curves (or, equivalently, weighted traces of products of intertwining operators) [B],[F], and the KZB equations with the Belavin r-matrix are satisfied by conformal blocks for the WZW model twisted by the rotation of the Dynkin diagram $[\mathrm{E}],[\mathrm{KT}]$. It is therefore natural to expect that a similar interpretation exists for all dynamical r-matrices.

In this paper, we propose such an interpretation. Namely, for any Kac-Moody algebra and a (nondegenerate) generalized BD triple we show that weighted traces of products of intertwining operators, "twisted" by $T$, satisfy the KZB equations with the corresponding dynamical r-matrix from [S].

We consider two cases: operators with values in representations from category $\mathcal{O}$, and operators with values in finite dimensional representations for affine Lie algebras. In the first case we get the KZB equations for trigonometric dynamical r-matrices corresponding to generalized Belavin-Drinfeld triples for Kac-Moody algebras, and in the second case we get the KZB equations for elliptic dynamical r-matrices which are intermediate between Felder's and Belavin's elliptic r-matrices.

In conclusion we would like to point out some directions of future research.

First of all, it turns out that classical dynamical r-matrices mentioned above can be explicitly quantized. To obtain such a quantization has been an open problem, except for a few special cases, but we will present a complete solution of this problem in our next paper (joint with Travis Schedler).

In another paper we plan to generalize the results of the present paper to quantum groups, following the ideas of [EV3], where it was done for the case $\Gamma_{1}=$ $\Gamma_{2}=\Gamma$ and $T=$ Id. This will give quantum KZB equations, which are difference equations involving quantum dynamical R-matrices which are quantizations of the classical dynamical r-matrices that appeared in this paper. In the case of the Belavin R-matrix, these equations are the elliptic qKZ equations for the 8-vertex model, which play an important role in statistical mechanics.

\section{Classical dynamical r-matrices for Kac-Moody algebras}

In $[\mathrm{S}]$ the second author associated a solution of the classical dynamical YangBaxter equation to every generalized Belavin-Drinfeld triple for simple Lie algebras. This construction easily extends to any Kac-Moody algebra. We recall this construction in this section.

Preliminaries. Let $A=\left(a_{i j}\right)$ be a symmetrizable generalized Cartan matrix of size $n$ and rank $l$. Let $(\mathfrak{h}, \Gamma, \check{\Gamma})$ be a realization of $A$, i.e., $\mathfrak{h}$ is a complex vector space of dimension $2 n-l, \Gamma=\left\{\alpha_{1}, \ldots \alpha_{n}\right\} \subset \mathfrak{h}^{*}$, and $\check{\Gamma}=\left\{h_{1}, \ldots h_{n}\right\} \subset \mathfrak{h}$ are linearly independent sets and $\left\langle\alpha_{j}, h_{i}\right\rangle=a_{i j}$. Let $\mathfrak{g}=\mathfrak{n}_{-} \oplus \mathfrak{h} \oplus \mathfrak{n}_{+}$be the KacMoody algebra associated to $A$, i.e., $\mathfrak{g}$ is generated by elements $e_{i}, f_{i}, i=1, \ldots n$ and $\mathfrak{h}$ with relations

$$
\left[e_{i}, f_{j}\right]=\delta_{i j} h_{i}, \quad[\mathfrak{h}, \mathfrak{h}]=0, \quad\left[h, e_{i}\right]=\left\langle\alpha_{i}, h\right\rangle e_{i}, \quad\left[h, f_{i}\right]=-\left\langle\alpha_{i}, h\right\rangle f_{i},
$$


together with the Serre relations (see $[K])$. Set $\mathfrak{b}_{ \pm}=\mathfrak{h} \oplus \mathfrak{n}_{ \pm}$. Let $\mathfrak{h}^{\prime}=\bigoplus \mathbb{C} h_{i}$ and let $($, ) be a nondegenerate invariant bilinear form on $\mathfrak{g}$. Recall that the restriction of $(,$,$) to \mathfrak{h}$ is nondegenerate (hence it defines a form on $\mathfrak{h}^{*}$, which we also denote by $()$,$) , and that the kernel of the restriction of ($,$) to \mathfrak{h}^{\prime}$ is equal to the center $\mathfrak{c}$ of $\mathfrak{g}$. Let $\Delta=\Delta^{+} \cup \Delta^{-} \subset \mathfrak{h}^{*}$ be the root system of $\mathfrak{g}$ and let $\mathfrak{g}_{\alpha}$ denote the weight subspace corresponding to a root $\alpha$. For each $\alpha \in \Delta^{+}$ fix bases $e_{\alpha}^{(1)}, \ldots e_{\alpha}^{\left(\operatorname{dim} \mathfrak{g}_{\alpha}\right)}$ and $f_{\alpha}^{(1)}, \ldots f_{\alpha}^{\left(\operatorname{dim} \mathfrak{g}_{\alpha}\right)}$ of $\mathfrak{g}_{\alpha}$ and $\mathfrak{g}_{-\alpha}$ respectively such that $\left(e_{\alpha}^{(i)}, f_{\alpha}^{(j)}\right)=\delta_{i j}$. The vector $\left[e_{\alpha}^{(i)}, f_{\alpha}^{(i)}\right]$ is independent of the choice of $i$ and we set $h_{\alpha}=\left[e_{\alpha}^{(i)}, f_{\alpha}^{(i)}\right]$ for any $i=1 \ldots \operatorname{dim} \mathfrak{g}_{\alpha}$. Let $\rho \in \mathfrak{h}^{*}$ be an element satisfying $\left(\rho, h_{i}\right)=1$ for all $i$. Identifying $\mathfrak{h}$ with $\mathfrak{h}^{*}$ via the form $($,$) , we can$ regard $\rho$ as an element of $\mathfrak{h}$ ( $\rho$ is well-defined up to adding a central element).

Definition. A generalized Belavin-Drinfeld triple is a triple $\left(\Gamma_{1}, \Gamma_{2}, T\right)$ where $\Gamma_{1}, \Gamma_{2} \subset \Gamma$ and $T: \Gamma_{1} \stackrel{\sim}{\rightarrow} \Gamma_{2}$ is an inner product preserving bijection.

Given a generalized Belavin-Drinfeld triple $\left(\Gamma_{1}, \Gamma_{2}, T\right)$, we let $\Gamma_{3}$ be the subset of $\Gamma_{1} \cap \Gamma_{2}$ consisting of roots which return to their original position after applying $T$ several times. It is clear that $\left(\Gamma_{1} \backslash \Gamma_{3}, \Gamma_{2} \backslash \Gamma_{3}, T\right)$ is an ordinary Belavin-Drinfeld triple (i.e. $T$ satisfies the nilpotency condition) and $\left(\Gamma_{3}, \Gamma_{3}, T\right)$ is generalized Belavin-Drinfeld triple on which $T$ is an automorphism of the Dynkin subdiagram $\Gamma_{3}$.

Set $\mathfrak{l}=\left(\sum_{\alpha \in \Gamma_{1}} \mathbb{C}(\alpha-T \alpha)\right)^{\perp} \subset \mathfrak{h}$ and let $\mathfrak{h}_{0} \subset \mathfrak{h}$ be the orthogonal complement of $\mathfrak{l}$ in $\mathfrak{h}$ with respect to the inner product on $\mathfrak{h}$.

Definition. We say that the generalized Belavin-Drinfeld triple is nondegenerate if the restriction of $($,$) to \mathfrak{l}$ is nondegenerate.

In the nondegenerate case, we have $\mathfrak{h}=\mathfrak{l} \oplus \mathfrak{h}_{0}$.

Note that every generalized Belavin-Drinfeld triple on a Dynkin diagram of finite type or a connected Dynkin diagram of affine type is nondegenerate. Indeed, in the finite type case, it is enough to assume that $\Gamma_{1} \cup \Gamma_{2}=\Gamma$, and the inner product is positive on the root lattice. Since $\mathfrak{l}$ is spanned by real linear combinations of roots, it is automatically nondegenerate. A similar argument works in the affine case. Nondegeneracy is also the case when $\Gamma_{1}=\Gamma_{2}$, because in this case $T$ gives rise to a finite order (hence semisimple) orthogonal automorphism of $\mathfrak{h}$, and $\mathfrak{l}=\mathfrak{h}^{T}$.

Let $\left(\Gamma_{1}, \Gamma_{2}, T\right)$ be a nondegenerate generalized Belavin-Drinfeld triple. It is convenient to choose an orthonormal basis $\left(x_{j}\right)_{j \in I}$ of $\mathfrak{h}$ with respect to $($,$) in$ such a way that

$$
\mathfrak{l}=\bigoplus_{j \in I_{1}} \mathbb{C} x_{j}, \quad \mathfrak{h}_{0}=\bigoplus_{j \in I_{2}} \mathbb{C} x_{j}
$$

for suitable disjoint subsets $I_{1}, I_{2} \subset I$ such that $I=I_{1} \cup I_{2}$.

Let $\mathfrak{h}_{i}$ be the subspaces of $\mathfrak{h}$ spanned by $h_{\alpha}, \alpha \in \Gamma_{i}$.

The following Lemma is straightforward but important for the considerations below. 
Lemma 2.1. There exists a unique Lie algebra homomorphism $B: \mathfrak{n}_{-} \oplus \mathfrak{h}_{1} \rightarrow$ $\mathfrak{n}_{-} \oplus \mathfrak{h}_{2}$ (resp. $\left.B^{-1}: \mathfrak{n}_{+} \oplus \mathfrak{h}_{2} \rightarrow \mathfrak{n}_{+} \oplus \mathfrak{h}_{1}\right)$ such that $B\left(f_{\alpha}\right)=f_{T(\alpha)}, B\left(h_{\alpha}\right)=h_{T(\alpha)}$, if $\alpha \in \Gamma_{1}, B\left(f_{\alpha}\right)=0$ if $\alpha \notin \Gamma_{1}$ (resp. $B^{-1}\left(e_{\alpha}\right)=e_{T^{-1}(\alpha)}, B^{-1}\left(h_{\alpha}\right)=h_{T^{-1}(\alpha)}$ if $\alpha \in \Gamma_{2}, B^{-1}\left(e_{\alpha}\right)=0$ if $\left.\alpha \notin \Gamma_{2}\right)$.

We will extend the homomorphisms $B, B^{-1}$ of Lie algebras to the corresponding homomorphisms of their universal enveloping algebras.

Let $\left\langle\Gamma_{i}\right\rangle, i \in\{1,2,3\}$ be the set of roots $\alpha \in \Delta^{+}$which are linear combinations of simple roots from $\Gamma_{i}$. Let $\mathfrak{g}_{\Gamma_{i}}$ be the subalgebra of $\mathfrak{g}$ generated by $\mathfrak{g}_{\alpha}, \mathfrak{g}_{-\alpha}$, $\alpha \in \Gamma_{i}$. The map $B$ restricts to an automorphism of $\mathfrak{g}_{\Gamma_{3}}$. For each $\alpha \in\left\langle\Gamma_{3}\right\rangle$, let $N_{\alpha}$ be the order of the action of $B$ on $\alpha$, i.e., $B^{N_{\alpha}} \alpha=\alpha$ but $B^{r} \alpha \neq \alpha$ for $1 \leq r<N_{\alpha}$. Finally, it will be convenient to assume that $f_{\alpha}^{(t)}$ and $e_{\alpha}^{(t)}$ are eigenvectors for $B^{N_{\alpha}}$, and set $B^{N_{\alpha}} f_{\alpha}^{(t)}=\theta_{\alpha}^{(t)} f_{\alpha}^{(t)}$. Note that $B^{N_{\alpha}} e_{\alpha}^{(t)}=\theta_{\alpha}^{(t)-1} e_{\alpha}^{(t)}$.

Finally, let us define the Cayley transform of $T$ on $\mathfrak{h}_{0}$. To do this, we need the following straightforward Lemma.

Lemma 2.2. For any $x \in \mathfrak{h}_{0}$, there exists a unique $y \in \mathfrak{h}_{0}$ such that for all $\alpha \in \Gamma_{1}$ one has $(\alpha-T \alpha, y)=(\alpha+T \alpha, x)$.

It is clear that $y$ depends linearly on $x$. We will write $y=C_{T} x$. It is easy to check that the operator $C_{T}: \mathfrak{h}_{0} \rightarrow \mathfrak{h}_{0}$ is skewsymmetric. It is called the Cayley transform of $T$.

Proposition 2.1 ([S]). The function $r_{T}: \mathfrak{l}^{*} \rightarrow(\mathfrak{g} \otimes \mathfrak{g})^{\mathfrak{l}}$

$$
\begin{aligned}
r_{T}(\lambda)=-\frac{1}{2} \sum_{j \in I} x_{j} \otimes x_{j}+\frac{1}{2} \sum_{i \in I_{2}} C_{T} x_{i} \otimes x_{i} & -\sum_{\alpha, t} f_{\alpha}^{(t)} \otimes e_{\alpha}^{(t)} \\
& +\sum_{\alpha, t} \sum_{l=1}^{\infty} e^{-l(\alpha, \lambda)} e_{\alpha}^{(t)} \wedge B^{l} f_{\alpha}^{(t)},
\end{aligned}
$$

is a solution of the classical dynamical Yang-Baxter equation

$$
\begin{aligned}
\sum_{i}\left(x_{i}^{(1)} \otimes \frac{\partial}{\partial x_{i}} r^{23}(\lambda)-x_{i}^{(2)} \frac{\partial}{\partial x_{i}} r^{13}(\lambda)+x_{i}^{(3)} \frac{\partial}{\partial x_{i}} r^{12}(\lambda)\right) \\
+\left[r^{12}(\lambda), r^{13}(\lambda)\right]+\left[r^{13}(\lambda), r^{23}(\lambda)\right]+\left[r^{12}(\lambda), r^{23}(\lambda)\right]=0 .
\end{aligned}
$$

Remarks. i) In the expression for $r_{T}(\lambda)$, the sum $\sum_{l=1}^{\infty} e^{-l(\alpha, \lambda)} e_{\alpha}^{(t)} \wedge B^{l} f_{\alpha}^{(t)}$ is finite if $\alpha \notin\left\langle\Gamma_{3}\right\rangle$ and is an infinite series convergent to a rational function of $e^{(\alpha, \lambda)}$ if $\alpha \in\left\langle\Gamma_{3}\right\rangle$.

Consider the special case when $\Gamma_{1}=\Gamma_{2}=\Gamma$, and $T$ is an automorphism of the Dynkin diagram. Let $N$ be the order of $B$ (i.e. $N$ is the smallest number divisible by all $N_{\alpha}$ such that $\left(\theta_{\alpha}^{(t)}\right)^{N / N_{\alpha}}=1$ ). In this case, the formula for $r_{T}(\lambda)$ 
can be written in the form

$$
\begin{aligned}
r_{T}(\lambda)=-\frac{1}{2} \sum_{j \in I} x_{j} \otimes x_{j}+\frac{1}{2} \sum_{i \in I_{2}} C_{T} x_{i} \otimes & x_{i}-\sum_{\alpha, t} f_{\alpha}^{(t)} \otimes e_{\alpha}^{(t)} \\
& +\sum_{\alpha, t} \sum_{l=1}^{N} \frac{e^{-l(\alpha, \lambda)}}{1-e^{-N(\alpha, \lambda)}} e_{\alpha}^{(t)} \wedge B^{l} f_{\alpha}^{(t)} .
\end{aligned}
$$

Examples. i) When $T=1$ one obtains Felder's trigonometric dynamical rmatrix

$$
r(\lambda)=-\frac{\Omega}{2}+\sum_{\alpha>0, t} \frac{1}{2} \operatorname{cotanh}\left(\frac{1}{2}(\alpha, \lambda)\right) e_{\alpha}^{(t)} \wedge f_{\alpha}^{(t)},
$$

where $\Omega=\sum_{\alpha, t}\left(e_{\alpha}^{(t)} \otimes f_{\alpha}^{(t)}+f_{\alpha}^{(t)} \otimes e_{\alpha}^{(t)}\right)+\sum_{i} x_{i} \otimes x_{i}$ is the Casimir element (see [EV1], Section 3).

ii) Consider $\mathfrak{g}=\operatorname{sl}(3)$ and let $T$ be the automorphism exchanging the two simple roots. Then $\mathfrak{l}=\mathbb{C} \rho$, so we can regard the element $\lambda \in \mathfrak{l}^{*}$ as a scalar. In this case, the dynamical r-matrix $r_{T}(\lambda)$ is

$$
\begin{aligned}
r_{T}(\lambda)=- & \frac{1}{2} \sum_{i \in I} x_{i} \otimes x_{i}-\frac{e^{-2 \lambda}}{1-e^{-2 \lambda}}\left(e_{\alpha_{1}} \otimes f_{\alpha_{1}}+e_{\alpha_{2}} \otimes f_{\alpha_{2}}\right) \\
- & \frac{e^{-2 \lambda}}{1+e^{-2 \lambda}} e_{\alpha_{1}+\alpha_{2}} \otimes f_{\alpha_{1}+\alpha_{2}}-\frac{1}{1-e^{-2 \lambda}}\left(f_{\alpha_{1}} \otimes e_{\alpha_{1}}+f_{\alpha_{2}} \otimes e_{\alpha_{2}}\right) \\
& \quad-\frac{1}{1+e^{-2 \lambda}} f_{\alpha_{1}+\alpha_{2}} \otimes e_{\alpha_{1}+\alpha_{2}}+\frac{e^{-\lambda}}{1-e^{-2 \lambda}}\left(e_{\alpha_{1}} \wedge f_{\alpha_{2}}+e_{\alpha_{2}} \wedge f_{\alpha_{1}}\right) .
\end{aligned}
$$

Note that in this example the eigenvalue $\theta_{\alpha_{1}+\alpha_{2}}^{(1)}$ of $B$ is -1 , which is the reason for the appearance of denominators $1+e^{-2 \lambda}$.

In the next section we give the representation-theoretic interpretation of the KZB equations associated to the dynamical r-matrix $r_{T}(\lambda)$.

\section{Traces of intertwining operators}

3.1. Traces. For any $\mathfrak{h}$-diagonalizable $\mathfrak{g}$-module $V$ let $V[\lambda]$ denote the subspace of $V$ of weight $\lambda \in \mathfrak{h}^{*}$. Let $M_{\lambda}$ be the Verma module of highest weight $\lambda$ and let $v_{\lambda} \in M_{\lambda}[\lambda]$ be a highest weight vector. Let $M_{\lambda}^{*}$ be the graded dual Verma module: $M_{\lambda}^{*}=\bigoplus_{\mu} M_{\lambda}[\mu]^{*}$ as a vector space and the Lie algebra $\mathfrak{g}$ acts by

$$
x . a(u)=-a(x . u) \quad \forall x \in \mathfrak{g}, u \in M_{\lambda}, a \in M_{\lambda}^{*} .
$$

Let $v_{\lambda}^{*} \in M_{\lambda}^{*}[-\lambda]$ be the lowest weight vector satisfying $\left\langle v_{\lambda}^{*}, v_{\lambda}\right\rangle=1$. Note that $M_{\lambda}$ and $M_{\lambda}^{*}$ are irreducible for generic values of $\lambda$.

Recall the definition of the quadratic Casimir operator $C$ :

$$
C=2 \rho+\sum_{j} x_{j}^{2}+2 \sum_{\alpha \in \Delta^{+}} \sum_{i} f_{\alpha}^{(i)} e_{\alpha}^{(i)} .
$$

The operator $C$ acts on $M_{\lambda}$ by multiplication by $\Delta_{\lambda}:=(\lambda, \lambda+2 \rho)$. 
Let $\lambda, \mu \in \mathfrak{h}^{*}$ and let $V$ be a $\mathfrak{g}$-module from the category $\mathcal{O}$. We will consider compositions of intertwining operators of the form

$$
\Phi: M_{\lambda} \rightarrow M_{\mu} \otimes V
$$

The following lemma is well known (a proof can be found e.g., in [EV2], [EFK1]) :

Lemma 3.1. Suppose that $M_{\mu}^{*}$ is irreducible. Then the map

$$
\operatorname{Hom}_{\mathfrak{g}}\left(M_{\lambda}, M_{\mu} \otimes V\right) \rightarrow V[\lambda-\mu], \quad \Phi \mapsto\left\langle v_{\mu}^{*}, \Phi v_{\lambda}\right\rangle,
$$

is an isomorphism.

Given $v \in V[\lambda-\mu]$, if $M_{\mu}^{*}$ is irreducible, we will denote by $\Phi_{\lambda}^{v}$ the unique intertwiner $M_{\lambda} \rightarrow M_{\mu} \otimes V$ satisfying $\left\langle v_{\mu}^{*}, \Phi v_{\lambda}\right\rangle=v$.

For any $\lambda \in \mathfrak{h}^{*}$ and any $\mathfrak{h}$-semisimple $\mathfrak{g}$-module $V$ we denote by $\lambda \in$ End $(V)$ the operator satisfying $\lambda_{\mid V[\nu]}=(\lambda, \nu)$.

Let $\mu, \mu^{\prime} \in \mathfrak{h}^{*}$. Consider the linear operator $B: M_{\mu^{\prime}} \rightarrow M_{\mu}$ defined by $B\left(x v_{\mu^{\prime}}\right)=B(x) v_{\mu}$ for any $x \in U \mathfrak{n}_{-}$. The following lemma is straightforward.

Lemma 3.2. Let $\mu, \mu^{\prime}$ be such that $\left(\mu^{\prime}, \alpha\right)=(\mu, T \alpha)$ for all $\alpha \in \Gamma_{1}$. Then for every $x \in U\left(\mathfrak{n}_{-} \oplus \mathfrak{h}_{1}\right)$ we have $B x=B(x) B$ and for every $x \in U\left(\mathfrak{n}_{+} \oplus \mathfrak{h}_{2}\right)$ we have $x B=B B^{-1}(x)$.

Let $V_{1}, V_{2}, \ldots, V_{r}$ be $\mathfrak{g}$-modules from the category $\mathcal{O}, v_{1} \in V_{1}, \ldots, v_{r} \in V_{r}$ homogeneous vectors of weights $\nu_{1}, \ldots, \nu_{r}$ respectively. Set $\nu=\sum \nu_{i}$ and consider (for generic $\mu$ ) the composition

$$
\Phi_{\mu-\nu_{2}-\ldots-\nu_{r}}^{v_{1}} \cdots \Phi_{\mu}^{v_{r}} B e^{\lambda}: M_{\mu^{\prime}} \rightarrow M_{\mu-\nu} \otimes V_{1} \otimes \cdots \otimes V_{r}
$$

where $\lambda \in \mathfrak{l}^{*}, \mu^{\prime} \in \mathfrak{h}^{*},\left(\mu^{\prime}, \alpha\right)=(\mu, T \alpha)$ for $\alpha \in \Gamma_{1}$ (we regard $\mathfrak{l}^{*}$ as a subspace of $\mathfrak{h}^{*}$ using the inner product).

If

$$
\mu^{\prime}=\mu-\nu
$$

we can define

$$
F^{v_{1}, \ldots, v_{r}}(\lambda, \mu)=\operatorname{Tr}\left(\Phi_{\mu-\nu_{2}-\ldots-\nu_{r}}^{v_{1}} \cdots \Phi_{\mu}^{v_{r}} B e^{\lambda}\right):\left(\mathfrak{h}^{*}\right)^{B} \rightarrow V_{1} \otimes \cdots \otimes V_{r} .
$$

If $\nu \in \mathfrak{l}^{\perp}$, the space of solutions of (3.2) in $\mu$ is an $\mathfrak{l}^{*}$-principal homogeneous space. Note that it follows from the Kac-Kazhdan conditions (see [KK]) and from the fact that $\rho \in \mathfrak{l}$ that for any fixed $v_{1}, \ldots, v_{r}$ the composition (3.1) is defined for generic values of $\mu$ in any $\mathfrak{l}^{*}$-principal homogeneous space. In particular, $F^{v_{1}, \ldots, v_{r}}(\lambda, \mu)$ is a formal series in $\lambda$ whose coefficients are trigonometric functions of $\mu$ with values in the space $\left(V_{1} \otimes \cdots \otimes V_{r}\right)^{\mathfrak{l}}$. 
Set

$$
\delta_{B}(\lambda)=\left(\operatorname{Tr}_{\mid M_{-\rho}}\left(B e^{\lambda}\right)\right)^{-1}
$$

(a twisted version of the Weyl denominator).

Lemma 3.3. We have $\delta_{B}(\lambda)=e^{(\rho, \lambda)} \prod_{\bar{\alpha} \in\left\langle\Gamma_{3}\right\rangle / B} \prod_{t}\left(1-\theta_{\alpha}^{(t)} e^{-N_{\alpha}(\alpha, \lambda)}\right)$.

Proof. Identify $U \mathfrak{n}^{-}$with $M_{-\rho}$ by $u \mapsto u v_{-\rho}$. Let $\mathbb{C}=U_{0} \subset U_{1} \subset \ldots$ denote the canonical filtration of $U \mathfrak{n}^{-}$. Since $\left(U_{n}\right)$ is stable under $B e^{\lambda}$, we can replace $U \mathfrak{n}^{-}$by its associated graded algebra when computing $\operatorname{Tr}_{\mid U \mathfrak{n}^{-}}\left(B e^{\lambda}\right)$. By the PBW theorem, Gr $U \mathfrak{n}^{-}=S \mathfrak{n}^{-}$. By definition $B$ acts nilpotently on $f_{\alpha}^{(t)}$ unless $\alpha \in\left\langle\Gamma_{3}\right\rangle$. Hence $\operatorname{Tr}_{\mid S \mathfrak{n}^{-}}\left(B e^{\lambda}\right)=\operatorname{Tr}_{\mid S \mathfrak{n}_{\Gamma_{3}}^{-}}\left(B e^{\lambda}\right)$ where $\mathfrak{n}_{\Gamma_{3}}^{-}=\mathfrak{n}^{-} \cap \mathfrak{g}_{\Gamma_{3}}$. The Lemma now follows from

$$
S \mathfrak{n}_{\Gamma_{3}}^{-}=\bigotimes_{\bar{\alpha} \in\left\langle\Gamma_{3}\right\rangle / B, t} S\left(\mathbb{C} f_{\alpha}^{(t)} \oplus \ldots \oplus \mathbb{C} B^{N_{\alpha}-1} f_{\alpha}^{(t)}\right),
$$

and

$$
\operatorname{Tr}_{\mid S\left(\mathbb{C} f_{\alpha}^{(t)} \oplus \ldots \oplus \mathbb{C} B^{N_{\alpha}-1} f_{\alpha}^{(t)}\right)}\left(B e^{\lambda}\right)=\frac{1}{1-\theta_{\alpha}^{(t)} e^{-N_{\alpha}(\alpha, \lambda)}}
$$

We put $\mathbb{F}^{v_{1}, \ldots, v_{r}}(\lambda, \mu)=\delta_{B}(\lambda) F^{v_{1}, \ldots, v_{r}}(\lambda, \mu)$. This function is the main object of this paper.

3.2. The KZB equations for traces. The following theorem is one of our main results.

Theorem 3.1. The function $\mathbb{F}^{v_{1}, \ldots, v_{r}}(\lambda, \mu)$ satisfies the following system of differential equations, for $i=1, \ldots r$ :

$$
\begin{aligned}
\left(\sum_{j \in I_{1}} x_{j \mid V_{i}} \frac{\partial}{\partial x_{j}}+\right. & \left.\sum_{j>i} r_{T}(\lambda)_{\mid V_{i} \otimes V_{j}}-\sum_{j<i} r_{T}(\lambda)_{\mid V_{j} \otimes V_{i}}\right) \mathbb{F}^{v_{1}, \ldots, v_{r}}(\lambda, \mu) \\
& =\frac{1}{2}\left(\Delta_{\mu-\nu_{i+1}-\ldots-\nu_{r}}-\Delta_{\mu-\nu_{i}-\ldots-\nu_{r}}\right) \mathbb{F}^{v_{1}, \ldots, v_{r}}(\lambda, \mu) .
\end{aligned}
$$

These equations are called the KZB equations (see $[\mathrm{F}]$ ).

Proof. For simplicity of notation we will write $\mu_{i}=\mu-\nu_{i+1}-\ldots-\nu_{r}$. We compute the function

$$
A(\lambda, \mu)=\operatorname{Tr}\left(\Phi_{\mu_{1}}^{v_{1}} \ldots \Phi_{\mu_{i-1}}^{v_{i-1}}\left(C_{\mid M_{\mu_{i-1}}} \Phi_{\mu_{i}}^{v_{i}}-\Phi_{\mu_{i}}^{v_{i}} C_{\mid M_{\mu_{i}}}\right) \Phi_{\mu_{i+1}}^{v_{i+1}} \ldots \Phi_{\mu}^{v_{r}} B e^{\lambda}\right)
$$

in two different ways. On one hand, we have

$$
A(\lambda, \mu)=\left(\Delta_{\mu_{i-1}}-\Delta_{\mu_{i}}\right) F^{v_{1}, \ldots, v_{r}}(\lambda, \mu) .
$$


On the other hand, using the relations

$$
\begin{aligned}
\left(f_{\alpha}^{(t)} e_{\alpha}^{(t)}\right)_{\mid M_{\mu_{i-1}}} \Phi_{\mu_{i}}^{v_{i}}-\Phi_{\mu_{i}}^{v_{i}}\left(f_{\alpha}^{(t)} e_{\alpha}^{(t)}\right)_{\mid M_{\mu_{i}}}= & -\left\{f_{\alpha \mid M_{\mu_{i-1}}}^{(t)} e_{\alpha \mid V_{i}}^{(t)}+e_{\alpha \mid M_{\mu_{i-1}}}^{(t)} f_{\alpha \mid V_{i}}^{(t)}+\left(f_{\alpha}^{(t)} e_{\alpha}^{(t)}\right) \mid V_{i}\right\} \Phi_{\mu_{i}}^{v_{i}}, \\
\rho_{\mid M_{\mu_{i-1}}} \Phi_{\mu_{i}}^{v_{i}}-\Phi_{\mu_{i}}^{v_{i}} \rho_{\mid M_{\mu_{i}}}= & -\rho_{\mid V_{i}} \Phi_{\mu_{i}}^{v_{i}}, \\
x_{j \mid M_{\mu_{i-1}}}^{2} \Phi_{\mu_{i}}^{v_{i}}-\Phi_{\mu_{i}}^{v_{i}} x_{j \mid M_{\mu_{i}}}^{2}= & -\left\{x_{j \mid V_{i}}^{2}+2 x_{j \mid M_{\mu_{i-1}}} x_{j \mid V_{i}}\right\} \Phi_{\mu_{i}}^{v_{i}},
\end{aligned}
$$

we deduce that

$$
\begin{aligned}
A(\lambda, \mu)=\left\{-\sum_{j} x_{j \mid V_{i}}^{2}-2 \sum_{\alpha, t}\left(f_{\alpha}^{(t)} e_{\alpha}^{(t)}\right)_{\mid V_{i}}-2 \rho_{\mid V_{i}}\right\} & F^{v_{1}, \ldots, v_{r}}(\lambda, \mu) \\
& +A_{1}(\lambda, \mu)+A_{2}(\lambda, \mu)+A_{3}(\lambda, \mu),
\end{aligned}
$$

where

$$
\begin{aligned}
& A_{1}(\lambda, \mu)=-2 \sum_{j \in I} x_{j \mid V_{i}} \operatorname{Tr}\left(\Phi_{\mu_{1}}^{v_{1}} \ldots \Phi_{\mu_{i-1}}^{v_{i-1}} x_{j \mid M_{\mu_{i-1}}} \Phi_{\mu_{i}}^{v_{i}} \ldots \Phi_{\mu}^{v_{r}} B e^{\lambda}\right) \\
& A_{2}(\lambda, \mu)=-2 \sum_{\alpha, t} e_{\alpha \mid V_{i}}^{(t)} \operatorname{Tr}\left(\Phi_{\mu_{1}}^{v_{1}} \ldots \Phi_{\mu_{i-1}}^{v_{i-1}} f_{\alpha \mid M_{\mu_{i-1}}}^{(t)} \Phi_{\mu_{i}}^{v_{i}} \ldots \Phi_{\mu}^{v_{r}} B e^{\lambda}\right) \\
& A_{3}(\lambda, \mu)=-2 \sum_{\alpha, t} f_{\alpha \mid V_{i}}^{(t)} \operatorname{Tr}\left(\Phi_{\mu_{1}}^{v_{1}} \ldots \Phi_{\mu_{i-1}}^{v_{i-1}} e_{\alpha \mid M_{\mu_{i-1}}(t)}^{(t)} \Phi_{\mu_{i}}^{v_{i}} \ldots \Phi_{\mu}^{v_{r}} B e^{\lambda}\right) .
\end{aligned}
$$

Writing $A_{1}$ as a sum of two equal terms:

$$
\begin{aligned}
A_{1}(\lambda, \mu)=-\sum_{j \in I} x_{j \mid V_{i}} \operatorname{Tr}\left(\Phi_{\mu_{1}}^{v_{1}} \ldots \Phi_{\mu_{i-1}}^{v_{i-1}} x_{j \mid M_{\mu_{i-1}}} \Phi_{\mu_{i}}^{v_{i}} \ldots \Phi_{\mu}^{v_{r}} B e^{\lambda}\right) \\
-\sum_{j \in I} x_{j \mid V_{i}} \operatorname{Tr}\left(\Phi_{\mu_{1}}^{v_{1}} \ldots \Phi_{\mu_{i-1}}^{v_{i-1}} x_{j \mid M_{\mu_{i-1}}} \Phi_{\mu_{i}}^{v_{i}} \ldots \Phi_{\mu}^{v_{r}} B e^{\lambda}\right),
\end{aligned}
$$

and using the intertwining properties $\Phi x_{j}=\left(x_{j} \otimes 1+1 \otimes x_{j}\right) \Phi$ and $\left(1 \otimes \Phi x_{j}\right)=$ $\Phi x_{j}-\left(x_{j} \otimes 1\right) \Phi$ repeatedly in the first and second term of $A_{1}(\lambda, \mu)$ respectively, we get

$$
\begin{aligned}
& A_{1}(\lambda, \mu)=-\sum_{j \in I} x_{j \mid V_{i}}\left(\sum_{t<i} x_{j \mid V_{t}}-\sum_{t \geq i} x_{j \mid V_{t}}\right) F^{v_{1}, \ldots, v_{r}}(\lambda, \mu) \\
& -\sum_{j \in I} x_{j \mid V_{i}}\left(\operatorname{Tr}\left(x_{j \mid M_{\mu_{0}}} \Phi_{\mu_{1}}^{v_{1}} \ldots \Phi_{\mu}^{v_{r}} B e^{\lambda}\right)+\operatorname{Tr}\left(\Phi_{\mu_{1}}^{v_{1}} \ldots \Phi_{\mu}^{v_{r}} x_{j \mid M_{\mu}} B e^{\lambda}\right)\right) .
\end{aligned}
$$

Now there are two cases to consider, depending on whether $j \in I_{1}$ or $j \in I_{2}$. In the first case, by the cyclicity of the trace, we have

$$
\begin{aligned}
\operatorname{Tr}\left(x_{j \mid M_{\mu_{0}}} \Phi_{\mu_{1}}^{v_{1}} \cdots \Phi_{\mu}^{v_{r}} B e^{\lambda}\right)+\operatorname{Tr}\left(\Phi_{\mu_{1}}^{v_{1}} \cdots \Phi_{\mu}^{v_{r}} x_{j \mid M_{\mu}} B e^{\lambda}\right)= \\
\operatorname{Tr}\left(\Phi_{\mu_{1}}^{v_{1}} \ldots \Phi_{\mu}^{v_{r}}\left(B x_{j}+x_{j}\right)_{\mid M_{\mu}} B e^{\lambda}\right),
\end{aligned}
$$


and

$$
\begin{aligned}
\operatorname{Tr}\left(\Phi_{\mu_{1}}^{v_{1}} \cdots \Phi_{\mu}^{v_{r}}\left(B x_{j}+x_{j}\right)_{\mid M_{\mu}} B e^{\lambda}\right) & =2 \operatorname{Tr}\left(\Phi_{\mu_{1}}^{v_{1}} \cdots \Phi_{\mu}^{v_{r}} x_{j \mid M_{\mu}} B e^{\lambda}\right) \\
& =2 \frac{\partial}{\partial x_{j}} F^{v_{1}, \ldots, v_{r}}(\lambda, \mu)
\end{aligned}
$$

where the differentiation is taken with respect to the parameter $\lambda$.

Let us now deal with the second case. It is easy to check using Lemma 3.2 that for any $x \in \mathfrak{l}^{\perp}$, one has

$$
\begin{aligned}
\operatorname{Tr}\left(x_{\mid M_{\mu_{0}}} \Phi_{\mu_{1}}^{v_{1}} \cdots \Phi_{\mu}^{v_{r}} B e^{\lambda}\right)+\operatorname{Tr}\left(\Phi_{\mu_{1}}^{v_{1}} \cdots\right. & \left.\Phi_{\mu}^{v_{r}} x_{\mid M_{\mu}} B e^{\lambda}\right) \\
& =-\sum_{i}\left(C_{T} x\right)_{i} \operatorname{Tr}\left(\Phi_{\mu_{1}}^{v_{1}} \cdots \Phi_{\mu}^{v_{r}} B e^{\lambda}\right) .
\end{aligned}
$$

Therefore, we obtain

$$
\begin{array}{r}
A_{1}(\lambda, \mu)=\left(-\sum_{t<i} x_{j \mid V_{i}} x_{j \mid V_{t}}+\sum_{t \geq i} x_{j \mid V_{i}} x_{j \mid V_{t}}-2 \sum_{j \in I_{1}} x_{j \mid V_{i}} \frac{\partial}{\partial x_{j}}\right. \\
\left.+\sum_{j \in I_{2}} x_{j \mid V_{i}} \sum_{l=1}^{r} C_{T} x_{j \mid V_{l}}\right) F^{v_{1}, \ldots, v_{r}}(\lambda, \mu) \\
=\left(-\sum_{t<i} x_{j \mid V_{i}} x_{j \mid V_{t}}+\sum_{t \geq i} x_{j \mid V_{i}} x_{j \mid V_{t}}-2 \sum_{j \in I_{1}} x_{j \mid V_{i}} \frac{\partial}{\partial x_{j}}\right. \\
\left.+\sum_{j \in I_{2}} x_{j \mid V_{i}} \sum_{l=1, l \neq i}^{r} C_{T} x_{j \mid V_{l}}\right) F^{v_{1}, \ldots, v_{r}}(\lambda, \mu),
\end{array}
$$

where in the last equality we used the skew-symmetry of $\sum_{j} C_{T} x_{j} \otimes x_{j}$ to get rid of terms with $i=l$.

We now compute $A_{2}(\lambda, \mu)$. Using the intertwining property

$$
\Phi f_{\alpha}=\left(1 \otimes f_{\alpha}+f_{\alpha} \otimes 1\right) \Phi
$$

we have

$$
\begin{aligned}
\operatorname{Tr} & \left(\Phi_{\mu_{1}}^{v_{1}} \cdots \Phi_{\mu_{i-1}}^{v_{i-1}} f_{\alpha \mid M_{\mu_{i-1}}}^{(t)} \cdots \Phi_{\mu}^{v_{r}} B e^{\lambda}\right) \\
& =\operatorname{Tr}\left(\Phi_{\mu_{1}}^{v_{1}} \cdots f_{\alpha \mid M_{\mu_{i-2}}}^{(t)} \Phi_{\mu_{i-1}}^{v_{i-1}} \cdots \Phi_{\mu}^{v_{r}} B e^{\lambda}\right)+f_{\alpha \mid V_{i-1}}^{(t)} F^{v_{1}, \ldots, v_{r}}(\lambda, \mu) \\
& =\operatorname{Tr}\left(f_{\alpha \mid M_{\mu_{0}}}^{(t)} \Phi_{\mu_{1}}^{v_{1}} \cdots \Phi_{\mu}^{v_{r}} B e^{\lambda}\right)+\left(f_{\alpha \mid V_{1}}^{(t)}+\cdots+f_{\alpha \mid V_{i-1}}^{(t)}\right) F^{v_{1}, \ldots, v_{r}}(\lambda, \mu) \\
& =e^{-(\alpha, \lambda)} \operatorname{Tr}\left(\Phi_{\mu_{1}}^{v_{1}} \cdots \Phi_{\mu}^{v_{r}} B f_{\alpha \mid M_{\mu^{\prime}}}^{(t)} e^{\lambda}\right)+\left(f_{\alpha \mid V_{1}}^{(t)}+\cdots+f_{\alpha \mid V_{i-1}}^{(t)}\right) F^{v_{1}, \ldots, v_{r}}(\lambda, \mu) .
\end{aligned}
$$


Hence by Lemma 3.2

$$
\begin{aligned}
& \operatorname{Tr}\left(\Phi_{\mu_{1}}^{v_{1}} \cdots \Phi_{\mu_{i-1}}^{v_{i-1}} f_{\alpha \mid M_{\mu_{i-1}}}^{(t)} \cdots \Phi_{\mu}^{v_{r}} B e^{\lambda}\right)= \\
& \quad\left(\sum_{j<i} \sum_{l=0}^{\infty} e^{-l(\alpha, \lambda)} B^{l} f_{\alpha \mid V_{j}}^{(t)}+\sum_{j \geq i} \sum_{l=1}^{\infty} e^{-l(\alpha, \lambda)} B^{l} f_{\alpha \mid V_{j}}^{(t)}\right) F^{v_{1}, \ldots, v_{r}}(\lambda, \mu)
\end{aligned}
$$

A similar computation (with $e_{\alpha}$ moving to the right) shows that

$$
\begin{aligned}
& \operatorname{Tr}\left(\Phi_{\mu_{1}}^{v_{1}} \cdots \Phi_{\mu_{i-1}}^{v_{i-1}} e_{\alpha \mid M_{\mu_{i-1}}}^{(t)} \cdots \Phi_{\mu}^{v_{r}} B e^{\lambda}\right)= \\
& -\left(\sum_{j<i} \sum_{l=1}^{\infty} e^{-l(\alpha, \lambda)} B^{-l} e_{\alpha \mid V_{j}}^{(t)}+\sum_{j \geq i} \sum_{l=0}^{\infty} e^{-l(\alpha, \lambda)} B^{-l} e_{\alpha \mid V_{j}}^{(t)}\right) F^{v_{1}, \ldots, v_{r}}(\lambda, \mu) .
\end{aligned}
$$

Adding (3.9) and (3.10) and using the relation

$$
\left[e_{\alpha}^{(t)}, B^{l} f_{\alpha}^{(t)}\right]= \begin{cases}\left(\theta_{\alpha}^{(t)}\right)^{l / N_{\alpha}} h_{\alpha} & \text { if } \alpha \in\left\langle\Gamma_{3}\right\rangle \text { and } N_{\alpha} \mid l \\ 0 & \text { else }\end{cases}
$$

we get

$$
\begin{aligned}
& A_{2}(\lambda, \mu)+A_{3}(\lambda, \mu)-2 \sum_{\alpha, t}\left(f_{\alpha}^{(t)} e_{\alpha}^{(t)}\right)_{V_{i}} F^{v_{1}, \ldots v_{r}}(\lambda, \mu)= \\
& -2\left(\sum_{\alpha, t} \sum_{j<i} e_{\alpha \mid V_{i}}^{(t)}\left(T_{1}(\alpha)\right)_{\mid V_{j}}+\right. \\
& \left.\sum_{\alpha, t} \sum_{j>i} e_{\alpha \mid V_{i}}^{(t)}\left(T_{2}(\alpha)\right)_{\mid V_{j}}\right) F^{v_{1}, \ldots, v_{r}}(\lambda, \mu) \\
& +2\left(\sum_{\alpha, t} \sum_{j<i} f_{\alpha \mid V_{i}}^{(t)}\left(T_{3}(\alpha)\right)_{\mid V_{j}} F^{v_{1}, \ldots, v_{r}}(\lambda, \mu)+\right. \\
& \left.\sum_{\alpha, t} \sum_{j>i} f_{\alpha \mid V_{i}}^{(t)}\left(T_{4}(\alpha)\right)_{\mid V_{j}}\right) F^{v_{1}, \ldots, v_{r}}(\lambda, \mu) \\
& -2 \sum_{\alpha \in\left\langle\Gamma_{3}\right\rangle, t} \frac{1-\theta_{\alpha}^{(t)} e^{-N_{\alpha}(\alpha, \lambda)}}{h_{\alpha \mid V_{i}} F^{v_{1}, \ldots, v_{r}}(\lambda, \mu) .}
\end{aligned}
$$

where

$$
\begin{aligned}
T_{1}(\alpha)=\sum_{l=0}^{\infty} e^{-l(\alpha, \lambda)} B^{l} f_{\alpha}^{(t)}, & T_{2}(\alpha)=\sum_{l=1}^{\infty} e^{-l(\alpha, \lambda)} B^{l} f_{\alpha}^{(t)} \\
T_{3}(\alpha)=\sum_{l=1}^{\infty} e^{-l(\alpha, \lambda)} B^{-l} e_{\alpha}^{(t)}, & T_{4}(\alpha)=\sum_{l=0}^{\infty} e^{-l(\alpha, \lambda)} B^{-l} e_{\alpha}^{(t)} .
\end{aligned}
$$


Combining (3.4), (3.7) and (3.12) now gives the following relation for $F^{v_{1}, \ldots, v_{r}}(\lambda, \mu)$ :

$$
\begin{aligned}
&\left(\sum_{j \in I_{1}}\left(x_{j}\right)_{\mid V_{i}} \otimes \frac{\partial}{\partial x_{j}}+\rho_{\mid V_{i}}-K(\lambda)_{\mid V_{i}}\right. \\
&\left.+\sum_{j>i} r_{T}(\lambda)_{\mid V_{i} \otimes V_{j}}-\sum_{j<i} r_{T}(\lambda)_{\mid V_{j} \otimes V_{i}}\right) F^{v_{1}, \ldots, v_{r}}(\lambda, \mu) \\
& \quad=\frac{1}{2}\left(\Delta_{\mu-\nu_{i+1}-\ldots-\nu_{r}}-\Delta_{\mu-\nu_{i}-\ldots-\nu_{r}}\right) F^{v_{1}, \ldots, v_{r}}(\lambda, \mu)
\end{aligned}
$$

where

$$
K(\lambda)=\sum_{\alpha \in\left\langle\Gamma_{3}\right\rangle, t} \frac{1}{1-\theta_{\alpha}^{(t)} e^{-N_{\alpha}(\alpha, \lambda)}} h_{\alpha} .
$$

A direct computation shows that

$$
\sum_{j \in I_{1}} x_{j} \frac{\partial}{\partial x_{j}} \operatorname{Tr}_{\mid M_{-\rho}}\left(B e^{\lambda}\right)+(\rho-K(\lambda)) \operatorname{Tr}_{\mid M_{-\rho}}\left(B e^{\lambda}\right)=0 .
$$

It is easy to deduce (3.3) from the above equations.

\subsection{The second order equation for traces.}

Theorem 3.2. The function $\mathbb{F}^{v_{1}, \ldots, v_{r}}(\lambda, \mu)$ satisfies the following second order differential equation:

$$
\left(\sum_{j \in I_{1}} \frac{\partial^{2}}{\partial x_{j}^{2}}-\sum_{l, n=1}^{r} S_{T}(\lambda)_{\mid V_{l} \otimes V_{n}}\right)=(\mu+\rho, \mu+\rho) \mathbb{F}^{v_{1}, \ldots, v_{r}}(\lambda, \mu),
$$

where

$$
\begin{array}{r}
S_{T}(\lambda)=\sum_{\alpha, t} \sum_{s=0}^{\infty} \sum_{v=1}^{\infty} e^{-(s+v)(\alpha, \lambda)}\left(B^{s} f_{\alpha}^{(t)} \otimes B^{-v} e_{\alpha}^{(t)}+B^{-v} e_{\alpha}^{(t)} \otimes B^{s} f_{\alpha}^{(t)}\right) \\
-\sum_{j \in I_{2}} \frac{1-C_{T}}{2} x_{j} \otimes \frac{1-C_{T}}{2} x_{j} .
\end{array}
$$

Proof. Consider

$$
A^{\prime}(\lambda, \mu)=\operatorname{Tr}\left(\Phi_{\mu_{1}}^{v_{1}} \ldots \Phi_{\mu}^{v_{r}} C_{\mid M_{\mu}} B e^{\lambda}\right) .
$$

On one hand, we have

$$
A^{\prime}(\lambda, \mu)=\Delta_{\mu} F^{v_{1}, \ldots, v_{r}}(\lambda, \mu),
$$

and on the other hand,

$$
\begin{aligned}
A^{\prime}(\lambda, \mu) & =\operatorname{Tr}\left(\Phi_{\mu_{1}}^{v_{1}} \ldots \Phi_{\mu}^{v_{r}}\left(\sum_{j \in I} x_{j}^{2}+2 \rho+2 \sum_{\alpha, t} f_{\alpha}^{(t)} e_{\alpha}^{(t)}\right)_{\mid M_{\mu}} B e^{\lambda}\right) \\
& =A_{1}^{\prime}(\lambda, \mu)+A_{2}^{\prime}(\lambda, \mu)+A_{3}^{\prime}(\lambda, \mu)
\end{aligned}
$$


where

$$
\begin{aligned}
& A_{1}^{\prime}(\lambda, \mu)=\sum_{j \in I} \operatorname{Tr}\left(\Phi_{\mu_{1}}^{v_{1}} \ldots \Phi_{\mu}^{v_{r}} x_{j \mid M_{\mu}}^{2} B e^{\lambda}\right) \\
& A_{2}^{\prime}(\lambda, \mu)=2 \operatorname{Tr}\left(\Phi_{\mu_{1}}^{v_{1}} \ldots \Phi_{\mu}^{v_{r}} \rho_{\mid M_{\mu}} B e^{\lambda}\right)=2 \frac{\partial}{\partial \rho} F^{v_{1}, \ldots, v_{r}}(\lambda, \mu), \\
& A_{3}^{\prime}(\lambda, \mu)=2 \sum_{\alpha, t} \operatorname{Tr}\left(\Phi_{\mu_{1}}^{v_{1}} \ldots \Phi_{\mu}^{v_{r}}\left(f_{\alpha}^{(t)} e_{\alpha}^{(t)}\right)_{\mid M_{\mu}} B e^{\lambda}\right),
\end{aligned}
$$

where the differentiation in the second equation is taken with respect to $\lambda$. To compute $A_{1}^{\prime}(\lambda, \mu)$, note that, as in (3.5), (3.6),

$$
A_{1}^{\prime}(\lambda, \mu)=\sum_{j \in I_{1}} \frac{\partial^{2}}{\partial x_{j}^{2}} F^{v_{1}, \ldots, v_{r}}(\lambda, \mu)+\sum_{j \in I_{2}} \operatorname{Tr}\left(\Phi_{\mu_{r-1}}^{v_{1}} \ldots \Phi_{\mu}^{v_{r}} x_{j \mid M_{\mu}}^{2} B e^{\lambda}\right)
$$

The second term on the r.h.s of (3.15) can be evaluated by the same method as in the derivation of the KZB equations: for all $j \in I_{2}$ we have

$$
\operatorname{Tr}\left(\Phi_{\mu_{1}}^{v_{1}} \ldots \Phi_{\mu}^{v_{r}} x_{j \mid M_{\mu}}^{2} B e^{\lambda}\right)=\sum_{l, s} \frac{1-C_{T}}{2} x_{j \mid V_{l}} \frac{1-C_{T}}{2} x_{j \mid V_{s}} F^{v_{1}, \ldots, v_{r}}(\lambda, \mu) .
$$

We now compute $A_{3}^{\prime}(\lambda, \mu)$. We consider two cases

Case 1: $\alpha \notin\left\langle\Gamma_{3}\right\rangle$. By the intertwining property and the cyclicity of the trace again, we have

$$
\begin{aligned}
& \operatorname{Tr}\left(\Phi_{\mu_{1}}^{v_{1}} \ldots \Phi_{\mu}^{v_{r}}\left(f_{\alpha}^{(t)} e_{\alpha}^{(t)}\right)_{\mid M_{\mu}} B e^{\lambda}\right) \\
& =\sum_{l=1}^{r} f_{\alpha \mid V_{l}}^{(t)} \operatorname{Tr}\left(\Phi_{\mu_{1}}^{v_{1}} \ldots \Phi_{\mu}^{v_{r}} e_{\alpha \mid M_{\mu}}^{(t)} B e^{\lambda}\right) \\
& \quad+e^{-(\alpha, \lambda)} \operatorname{Tr}\left(\Phi_{\mu_{1}}^{v_{1}} \ldots \Phi_{\mu}^{v_{r}}\left(e_{\alpha}^{(t)} B\left(f_{\alpha}^{(t)}\right)\right)_{\mid M_{\mu}} B e^{\lambda}\right)
\end{aligned}
$$

Applying this equation repeatedly and using the relation (3.11), we obtain

$$
\begin{aligned}
& \operatorname{Tr}\left(\Phi_{\mu_{1}}^{v_{1}} \ldots \Phi_{\mu}^{v_{r}}\left(f_{\alpha}^{(t)} e_{\alpha}^{(t)}\right)_{\mid M_{\mu}} B e^{\lambda}\right)= \\
& \sum_{l=1}^{r} \sum_{s=0}^{\infty} e^{-s(\alpha, \lambda)} B^{s}\left(f_{\alpha}\right)_{\mid V_{l}} \operatorname{Tr}\left(\Phi_{\mu_{1}}^{v_{1}} \ldots \Phi_{\mu}^{v_{r}} e_{\alpha \mid M_{\mu}}^{(t)} B e^{\lambda}\right)
\end{aligned}
$$


Case 2: $\alpha \in\left\langle\Gamma_{3}\right\rangle$. Applying (3.17) $N_{\alpha}$ times yields

$$
\begin{gathered}
\operatorname{Tr}\left(\Phi_{\mu_{1}}^{v_{1}} \ldots \Phi_{\mu}^{v_{r}}\left(f_{\alpha}^{(t)} e_{\alpha}^{(t)}\right)_{\mid M_{\mu}} B e^{\lambda}\right)= \\
\sum_{l=1}^{r} \sum_{s=0}^{N_{\alpha}-1} e^{-s(\alpha, \lambda)} B^{s}\left(f_{\alpha}\right)_{\mid V_{l}} \operatorname{Tr}\left(\Phi_{\mu_{1}}^{v_{1}} \ldots \Phi_{\mu}^{v_{r}} e_{\alpha \mid M_{\mu}}^{(t)} B e^{\lambda}\right) \\
\quad+e^{-N_{\alpha}(\alpha, \lambda)} \theta_{\alpha}^{(t)} \operatorname{Tr}\left(\Phi_{\mu_{1}}^{v_{1}} \ldots \Phi_{\mu}^{v_{r}} h_{\alpha \mid M_{\mu}} B e^{\lambda}\right) \\
\quad+e^{-N_{\alpha}(\alpha, \lambda)} \theta_{\alpha}^{(t)} \operatorname{Tr}\left(\Phi_{\mu_{1}}^{v_{1}} \ldots \Phi_{\mu}^{v_{r}}\left(f_{\alpha}^{(t)} e_{\alpha}^{(t)}\right)_{\mid M_{\mu}} B e^{\lambda}\right),
\end{gathered}
$$

from which it follows that

$$
\begin{aligned}
& \text { 19) } \operatorname{Tr}\left(\Phi_{\mu_{1}}^{v_{1}} \ldots \Phi_{\mu}^{v_{r}}\left(f_{\alpha}^{(t)} e_{\alpha}^{(t)}\right)_{\mid M_{\mu}} B e^{\lambda}\right)= \\
& \frac{1}{1-\theta_{\alpha}^{(t)} e^{-N_{\alpha}(\alpha, \lambda)}}\left\{\sum_{l=1}^{r} \sum_{s=0}^{N_{\alpha}-1} e^{-s(\alpha, \lambda)} B^{s}\left(f_{\alpha}\right)_{\mid V_{l}} \operatorname{Tr}\left(\Phi_{\mu_{1}}^{v_{1}} \ldots \Phi_{\mu}^{v_{r}} e_{\alpha \mid M_{\mu}}^{(t)} B e^{\lambda}\right)\right. \\
& \left.\quad+\theta_{\alpha}^{(t)} e^{-N_{\alpha}(\alpha, \lambda)} \operatorname{Tr}\left(\Phi_{\mu_{1}}^{v_{1}} \ldots \Phi_{\mu}^{v_{r}} h_{\alpha \mid M_{\mu}} B e^{\lambda}\right)\right\} .
\end{aligned}
$$

The formula for $A_{3}^{\prime}(\lambda, \mu)$ now follows from (3.10). Equations (3.15),(3.16), (3.19) imply the following second-order differential equation for $F^{v_{1}, \ldots, v_{r}}(\lambda, \mu)$ :

$$
\begin{aligned}
\left(\frac{1}{2} \sum_{j \in I_{1}} \frac{\partial^{2}}{\partial x_{j}^{2}}+\frac{\partial}{\partial \rho}+H(\lambda)\right) & F^{v_{1}, \ldots, v_{r}}(\lambda, \mu)= \\
& \frac{1}{2}\left(\Delta_{\mu}+\sum_{l, n=1}^{r} S_{T}(\lambda)_{V_{l} \otimes V_{n}}\right) F^{v_{1}, \ldots, v_{r}}(\lambda, \mu)
\end{aligned}
$$

where

$$
H(\lambda)=\sum_{\alpha \in\left\langle\Gamma_{3}\right\rangle, t} \frac{1}{1-\theta_{\alpha}^{(t)} e^{-N_{\alpha}(\alpha, \lambda)}} \frac{\partial}{\partial h_{\alpha}} .
$$

In particular,

$$
\left(\frac{1}{2} \sum_{j \in I_{1}} \frac{\partial^{2}}{\partial x_{j}^{2}}+\frac{\partial}{\partial \rho}+H(\lambda)\right) \operatorname{Tr}_{\mid M_{-\rho}}\left(B e^{\lambda}\right)=\frac{1}{2} \Delta_{\rho} \operatorname{Tr}_{\mid M_{-\rho}}\left(B e^{\lambda}\right)
$$

which, together with (3.13) and (3.20) yields (3.14).

\subsection{Diagonalization of the KZB and the second order operators.}

Denote by $K_{j}(\lambda)$ the differential operators appearing on the left hand side of the KZB equations, and by $D(\lambda)$ the second order operator appearing in Theorem 3.2. These are operators on the space of functions of $\lambda$ with values in $\left(V_{1} \otimes \ldots \otimes V_{r}\right)^{\mathfrak{l}}$. It is known $[\mathrm{F}]$ that $K_{j}$ commute with each other. Besides, it can be shown that the operators $K_{j}$ commute with $D$ (in fact, this is also clear 
from the discussion below). This gives rise to the problem of simultaneous diagonalization of these operators. More precisely, the problem can be formulated as follows.

Fix a weight $\nu \in \mathfrak{l}^{\perp}$. Fix a generic point $\xi \in \mathfrak{l}^{*}$, and consider the space of formal series

$$
W_{\nu, \xi}:=e^{(\lambda, \xi+\rho)}\left(V_{1} \otimes \ldots \otimes V_{r}\right)[\nu]\left[\left[e^{-\left(\alpha_{i}, \lambda\right)}\right]\right] .
$$

It is clear that the operators $K_{j}, D$ act naturally in this space and are upper triangular with respect to the natural ordering. The problem is to find a (topological) basis of $W_{\nu, \xi}$ in which these operators are diagonal.

The following proposition provides such a basis.

Let $\mathcal{B}_{i}$ be homogeneous bases of $V_{i}$, and $\mathcal{B}(\nu)$ be the set of collections $\left(v_{1}, \ldots, v_{r}\right)$ of vectors $v_{i} \in \mathcal{B}_{i}$ such that the sum of their weights is $\nu$.

Proposition 3.1. For generic $\xi$, the functions $\mathbb{F}^{v_{1}, . ., v_{r}}\left(\lambda, \frac{1+C_{T}}{2} \nu+\xi-\sum n_{i} \alpha_{i}\right)$, where $n_{i} \geq 0$, and $\left(v_{1}, \ldots, v_{r}\right)$ run through $\mathcal{B}$, form a common topological eigenbasis of the operators $K_{j}, D$ in the space $W_{\nu, \xi}$.

This proposition follows immediately from the theorems of this section: the fact that the listed functions form a basis is obvious, so the only thing to be shown is that they are eigenfunctions, which was shown above.

\subsection{Quantum integrable systems associated to generalized Belavin-} Drinfeld triples for simple Lie algebras. In the case when the Lie algebra $\mathfrak{g}$ is finite dimensional, one can define other differential operators which commute with $K_{j}, D$.

Namely, if $Z$ is any element of the center of $U(\mathfrak{g})$ then there exists a unique differential operator $D_{Z}$ on $\mathfrak{l}^{*}$ with values in $\operatorname{End}\left(\left(V_{1} \otimes \ldots \otimes V_{r}\right)\left[\mathfrak{l}^{\perp}\right]\right)$ such that

$$
D_{Z} \mathbb{F}=\delta_{B}(\lambda) \operatorname{Tr}\left(\Phi_{\mu_{1}}^{v_{1}} \ldots \Phi_{\mu}^{v_{r}} Z_{\mid M_{\mu}} B e^{\lambda}\right) .
$$

For example, $D_{C}=D-(\rho, \rho)$.

It is easy to see that $D_{Z_{1} Z_{2}}=D_{Z_{1}} D_{Z_{2}}$, so the operators $D_{Z}$ form a commutative algebra. It is clear that these operators also diagonalize in the basis of the previous section. Thus, we get a "quantum integrable system", whose eigenstates are the functions $\mathbb{F}^{v_{1}, . ., v_{r}}$.

In the special case $\Gamma_{i}=\Gamma, T=i d$, this system is a generalized trigonometric Calogero-Moser system considered in [EFK2].

\section{Classical dynamical r-matrices with spectral parameter}

Applying the construction of Section 2 to an (untwisted) affine Lie algebra $\hat{\mathfrak{g}}$ and using the evaluation map $\mathrm{ev}_{z}: \hat{\mathfrak{g}} \rightarrow \mathfrak{g}$, one can obtain solutions of the classical dynamical Yang-Baxter equation with spectral parameter. This is done as follows. 
Let $\mathfrak{g}$ be a simple complex Lie algebra, and let $\tilde{\mathfrak{g}}=\mathfrak{g}\left[t, t^{-1}\right] \oplus \mathbb{C} c \oplus \mathbb{C} \partial$ be the associated affine Kac-Moody algebra, where $c$ is the central element and $\partial$ is the grading element. The commutation relations in $\tilde{\mathfrak{g}}$ are:

$$
[c, \tilde{\mathfrak{g}}]=0, \quad\left[x t^{n}, y t^{m}\right]=[x, y] t^{n+m}+n \delta_{n,-m} c, \quad\left[\partial, x t^{n}\right]=n x t^{n}, \quad \forall x, y \in \mathfrak{g} .
$$

Recall that the Cartan subalgebra of $\tilde{\mathfrak{g}}$ is $\tilde{\mathfrak{h}}=\mathfrak{h} \oplus \mathbb{C} c \oplus \mathbb{C} \partial$. Then $\tilde{\mathfrak{h}}^{*}=$ $\mathfrak{h}^{*} \oplus \mathbb{C} \Lambda_{0} \oplus \mathbb{C} \delta$ where $\Lambda_{0}, \delta$ are defined by $\left\langle\Lambda_{0}, \mathfrak{h}\right\rangle=\left\langle\Lambda_{0}, \partial\right\rangle=\langle\delta, \mathfrak{h}\rangle=\langle\delta, c\rangle=0$ and $\langle\delta, \partial\rangle=\left\langle\Lambda_{0}, c\right\rangle=1$. Under the standard bilinear form on $\tilde{\mathfrak{h}}, c$ and $\partial$ are orthogonal to $\mathfrak{h}$ and we have $(c, \partial)=1,(c, c)=(\partial, \partial)=0$.

The root system of $\tilde{\mathfrak{g}}$ is $\tilde{\Delta}=(\Delta+\mathbb{Z} \delta) \cup \mathbb{Z}^{*} \delta$. The root subspace corresponding to $\alpha+k \delta$ is spanned by $e_{\alpha} t^{k}$ if $\alpha \in \Delta^{+}, f_{-\alpha} t^{k}$ if $\alpha \in-\Delta^{+}$and equals $\mathfrak{h} t^{k}$ if $\alpha=0$. The system of positive roots is $\tilde{\Delta}^{+}=\Delta^{+} \cup \mathbb{N} \delta \cup(\Delta+\mathbb{N} \delta)$.

We will consider a twisted realization of the affine Lie algebra $\tilde{\mathfrak{g}}$. Let $g$ be the dual Coxeter number of $\mathfrak{g}$ and set $\epsilon=e^{\frac{2 i \pi}{g}}$. Consider the automorphism $\gamma=A d\left(e^{2 i \pi \rho / g}\right)$ of $\mathfrak{g}$. We have $\gamma\left(e_{\alpha}\right)=\epsilon^{|\alpha|} e_{\alpha}, \gamma\left(f_{\alpha}\right)=\epsilon^{-|\alpha|} f_{\alpha}$, and $\gamma_{\mid \mathfrak{h}}=$ $I d$. Let $\tilde{\mathfrak{g}}_{\gamma}$ be the subalgebra of $\tilde{\mathfrak{g}}$ consisting of all elements $a(t)+\lambda c+\mu \partial$ satisfying $a(\epsilon t)=\gamma(a(t))$. The elements $e_{\alpha} t^{|\alpha|+m g}, f_{\alpha} t^{-|\alpha|+m g}, x_{i} t^{m g}, c, \partial$ for $\alpha \in \Delta^{+}, m \in \mathbb{Z}$ and $\left(x_{i}\right)$ an orthonormal basis of $\mathfrak{h}$, form a $\mathbb{C}$-basis of $\tilde{\mathfrak{g}}_{\gamma}$. The proof of the following lemma is straightforward.

Lemma 4.1. The map $\phi: \tilde{\mathfrak{g}}_{\gamma} \rightarrow \tilde{\mathfrak{g}}$ defined by

$$
\begin{aligned}
& \phi\left(e_{\alpha} t^{|\alpha|+m g}\right)=e_{\alpha} t^{m}, \quad \phi\left(f_{\alpha} t^{-|\alpha|+m g}\right)=f_{\alpha} t^{m}, \quad \phi(\partial)=g \partial+\rho \\
& \phi\left(x_{i} t^{m g}\right)=x_{i} t^{m}, \quad(m \neq 0), \quad \phi\left(x_{i}\right)=x_{i}-\left(\rho, x_{i}\right) \frac{c}{g}, \quad \phi(c)=\frac{c}{g},
\end{aligned}
$$

is a Lie algebra isomorphism.

Let $\left(\Gamma_{1}, \Gamma_{2}, \tau\right)$ be a generalized Belavin-Drinfeld triple for $\hat{\mathfrak{g}}_{\gamma}$. Let $\tilde{\mathfrak{l}}$ be the subalgebra of $\tilde{\mathfrak{h}}$ of elements $x$ such that $(\alpha, x)=(T \alpha, x)$ for $\alpha \in \Gamma_{1}$. Let $\mathfrak{l}=\tilde{\mathfrak{l}} \cap \mathfrak{h}$. It is clear that $\tilde{\mathfrak{l}}$ contains $c$ and $\partial$, so $\tilde{\mathfrak{l}}=\mathbb{C} c \oplus \mathbb{C} \partial \oplus \mathfrak{l}$.

For any $z \in \mathbb{C}^{*}$ let $\mathrm{ev}_{z}: \mathfrak{g}\left[t, t^{-1}\right] \rightarrow \mathfrak{g}$ be the evaluation map defined by $\mathrm{ev}_{z}\left(x t^{n}\right)=z^{n} x$ for all $x \in \mathfrak{g}, n \in \mathbb{Z}$.

Fix a complex number $\tau$ with positive imaginary part. Let $\tilde{\lambda}=\lambda+2 \pi i \tau \delta / g$. Define

$$
\bar{r}_{T}(\lambda, z)=\left(\mathrm{ev}_{z} \otimes \mathrm{ev}_{1}\right)\left(\phi^{-1} \otimes \phi^{-1}\right)\left(r_{T}(\tilde{\lambda})\right),
$$

where $z \in \mathbb{C}^{*}, \lambda \in \mathfrak{l}^{*}$.

Remark. Although the evaluation maps are not defined on $\partial$, this definition makes sense, since $\partial$ occurs in $r_{T}$ in a combination $c \otimes \partial+\partial \otimes c$, and $\operatorname{ev}_{z}(c)=0$ for any $z$.

It is clear that $\bar{r}_{T}$ is a Laurent series in $z$ whose coefficients are meromorphic functions on $\mathfrak{l}^{*}$.

Proposition 4.1. The series $\bar{r}_{T}(\lambda, z): \mathfrak{l}^{*} \rightarrow(\mathfrak{g} \otimes \mathfrak{g})^{\mathfrak{l}}$ is convergent in a nonempty annulus, and extends to a meromorphic function on $\mathfrak{l}^{*} \times \mathbb{C}^{*}$. Moreover, this 
meromorphic function satisfies the dynamical Yang-Baxter equation with spectral parameter:

$$
\begin{aligned}
& \sum_{i} x_{i}^{(1)} \frac{\partial}{\partial x_{i}} r^{23}\left(\lambda, \frac{z_{2}}{z_{3}}\right)-x_{i}^{(2)} \frac{\partial}{\partial x_{i}} r^{13}\left(\lambda, \frac{z_{1}}{z_{3}}\right)+x_{i}^{(3)} \frac{\partial}{\partial x_{i}} r^{12}\left(\lambda, \frac{z_{1}}{z_{2}}\right) \\
&+\left[r^{12}\left(\lambda, \frac{z_{1}}{z_{2}}\right), r^{13}\left(\lambda, \frac{z_{1}}{z_{3}}\right)\right]+ {\left[r^{13}\left(\lambda, \frac{z_{1}}{z_{3}}\right), r^{23}\left(\lambda, \frac{z_{2}}{z_{3}}\right)\right] } \\
&+ {\left[r^{12}\left(\lambda, \frac{z_{1}}{z_{2}}\right), r^{23}\left(\lambda, \frac{z_{2}}{z_{3}}\right)\right]=0 }
\end{aligned}
$$

where $x_{i}$ is an orthonormal basis of $\mathfrak{h}$.

This proposition follows easily from Proposition 2.1.

Now we would like to compute $\bar{r}_{T}(\lambda, z)$ explicitly. For the sake of simplicity we will restrict ourselves to triples of the form $\left(\Gamma_{1}=\Gamma_{2}=\Gamma, T\right)$ (i.e $T$ is an automorphism of the Dynkin diagram): these triples give rise to elliptic dynamical r-matrices.

Remark. More general triples give rise to partially trigonometric and partially elliptic r-matrices.

Let $T$ be an automorphism of the Dynkin diagram $\Gamma$ of $\tilde{\mathfrak{g}}$ of order $N$. As in Section 2, let $B$ be the lift of $T$ to an automorphism of $\tilde{\mathfrak{g}}$ of order $N$. By Lemma $4.1, B$ defines an automorphism of $\tilde{\mathfrak{g}}_{\gamma}$. Note that the action of $B$ on $\tilde{\mathfrak{g}}_{\gamma}$ preserves the principal gradation, i.e the exists a unique automorphism $\beta$ of $\mathfrak{g}$ such that $B\left(x t^{m}\right)=\beta(x) t^{m}$ for any $x t^{m} \in \tilde{\mathfrak{g}}_{\gamma}$. Furthermore, $c$ and $\partial$ are $B$-invariant, and $\mathfrak{h}$ is $B$-stable. Like before, we will choose an orthonormal basis $x_{i}$ of $\mathfrak{h}$ which is compatible with $\mathfrak{l}=\mathfrak{h}^{B}$ and $\mathfrak{l}^{\perp}$.

Let

$$
\theta(u \mid \tau)=-\sum_{j=-\infty}^{\infty} e^{\pi i\left(j+\frac{1}{2}\right)^{2} \tau+2 \pi i\left(j+\frac{1}{2}\right)\left(u+\frac{1}{2}\right)},
$$

be the Jacobi theta function. For brevity we will not write the dependence on $\tau$ explicitly. Introduce the functions

$$
\sigma_{w}(u)=\frac{\theta(w-u) \theta^{\prime}(0)}{\theta(w) \theta(u)}, \quad \chi(u)=\frac{\theta^{\prime}(u)}{\theta(u)}
$$

where $\theta^{\prime}$ is the derivative of $\theta$.

Let $z=e^{2 \pi i u / g}$. 
Proposition 4.2. The function $\bar{r}_{T}(\lambda, z): \mathfrak{l}^{*} \times \mathbb{C}^{*} \rightarrow(\mathfrak{g} \otimes \mathfrak{g})^{\mathfrak{l}}$ is equal to

$$
\begin{aligned}
\bar{r}_{T}(\lambda, z) & = \\
& -\sum_{\alpha>0} \sum_{l=0}^{N-1} \frac{1}{2 \pi i} e^{-l(\alpha, \tilde{\lambda})+2 \pi i|\alpha| u / g} \sigma_{\frac{N}{2 \pi i}(\alpha, \tilde{\lambda})}(u-l \tau \mid N \tau) e_{\alpha} \otimes \beta^{l}\left(f_{\alpha}\right) \\
& -\sum_{\alpha>0} \sum_{l=0}^{N-1} \frac{1}{2 \pi i} e^{l(\alpha, \tilde{\lambda})-2 \pi i|\alpha| u / g} \sigma_{-\frac{N}{2 \pi i}(\alpha, \tilde{\lambda})}(u-l \tau \mid N \tau) f_{\alpha} \otimes \beta^{l}\left(e_{\alpha}\right) \\
- & \sum_{j \in I} \sum_{l=0}^{N-1}\left(\frac{1}{2} \delta_{l 0}+\frac{1}{2 \pi i} \chi(u-l \tau \mid N \tau)\right) x_{j} \otimes \beta^{l} x_{j}+\frac{1}{2} \sum_{j \in I_{2}} \frac{\beta+1}{\beta-1} x_{j} \otimes x_{j}
\end{aligned}
$$

(here $\alpha$ runs through positive roots of $\mathfrak{g}$ ).

The proof of this proposition is by a direct calculation.

Thus we see that the above construction gives a dynamical r-matrix with the number of dynamical parameters equal to $d-1$, where $d$ is the number of orbits of $T$ on the Dynkin diagram.

Examples. i) When $T=1$, this yields (up to a gauge transformation) Felder's elliptic r-matrix, as shown in [EV1], Section 4.6.

ii) Let $\mathfrak{g}=\operatorname{sl}(n)$ and let $T$ be the rotation of the Dynkin diagram by $2 \pi k / n$. Note that in this case (and in this case only) $d=1$ and we obtain a non-dynamical r-matrix with spectral parameter. It is easy to check that it is equal to Belavin's classical elliptic r-matrix with modulus $\tau$ (see $[\mathrm{BD}]$ ).

\section{Twisted traces of intertwiners for affine Lie algebras}

In this section, we apply the same procedure as in Section 3 in the case of an affine Lie algebra $\tilde{\mathfrak{g}}$, but we consider finite-dimensional (evaluation) modules $V_{i}$ rather than modules from the category $\mathcal{O}$. We keep the notations of Section 4.

The Lie algebra $\tilde{\mathfrak{g}}_{\gamma}$ has a triangular decomposition $\tilde{\mathfrak{g}}_{\gamma}=\tilde{\mathfrak{n}}_{\gamma}^{+} \oplus \tilde{\mathfrak{h}} \oplus \tilde{\mathfrak{n}}_{\gamma}^{-}$where $\tilde{\mathfrak{n}}_{\gamma}^{+}$ (resp. $\tilde{\mathfrak{n}}_{\gamma}^{-}$) is the subalgebra spanned by $e_{\alpha} t^{|\alpha|+m g}, m \geq 0, f_{\alpha} t^{-|\alpha|+m g}, x_{i} t^{m g}$, $m>0$ (resp. spanned by $f_{\alpha} t^{-|\alpha|+m g}, m \leq 0, e_{\alpha} t^{|\alpha|+m g}, x_{i} t^{m g}, m<0$ ). This decomposition allows us to define highest weight Verma modules and dual Verma modules in the usual way. We put $\tilde{\mu}=\mu+\frac{k}{g} \Lambda_{0}-\frac{g(\mu, \mu)}{2(k+g)} \delta$ and denote by $M_{\mu, k}$ the Verma module $M_{\tilde{\mu}}$.

Finally, we define evaluation representations of $\tilde{\mathfrak{g}}_{\gamma}$. Let $V$ be a finitedimensional highest weight $\mathfrak{g}$-module with highest weight vector $v_{0}$. For any $\Delta \in \mathbb{C}$ let $z^{-\Delta} V\left[z, z^{-1}\right]$ denote the $\tilde{\mathfrak{g}}$-evaluation module, with $\tilde{\mathfrak{g}}$-action given by

$$
x t^{n} \cdot(v P(z))=x v z^{n} P(z), \quad c .(v P(z))=0, \quad \partial .(v P(z))=v z \frac{d}{d z} P(z),
$$

for all $x \in \mathfrak{g}, v \in V, P(z) \in z^{-\Delta} \mathbb{C}\left[z, z^{-1}\right]$. Let $z^{-\Delta} V_{\gamma}\left[z, z^{-1}\right] \subset z^{-\Delta} V\left[z, z^{-1}\right]$ be the subspace of all (Laurent) polynomials $v(z)$ satisfying $v(\epsilon z)=e^{2 i \pi \rho / g} v(z)$. 
Then $z^{-\Delta} V_{\gamma}\left[z, z^{-1}\right]$ is a $\tilde{\mathfrak{g}}_{\gamma}$-module (which is isomorphic to the usual $\tilde{\mathfrak{g}}$-evaluation module). From now on, we simply write $z^{-\Delta} V\left[z^{ \pm 1}\right]$ for $z^{-\Delta} V_{\gamma}\left[z^{ \pm 1}\right]$.

Proposition 3.1 admits an analogue in this situation (see e.g., [EFK1],[E]):

Proposition 5.1. Let $\mu \in \mathfrak{h}^{*}$ and $k \in \mathbb{C}$ and let $V$ be a finite-dimensional $\mathfrak{g}$ module. Suppose that $M_{\mu, k}^{*}$ is irreducible. Then for each $v \in V[\lambda-\mu]$ there exists a unique $\tilde{\mathfrak{g}}_{\gamma}$ intertwiner

$$
\tilde{\Phi}_{\mu, k}^{v}(z): M_{\lambda, k} \rightarrow M_{\mu, k} \hat{\otimes} z^{-\Delta} V\left[z^{ \pm} 1\right],
$$

where $\Delta=g \frac{(\lambda, \lambda)-(\mu, \mu)}{2(k+g)}$ such that

$$
\left\langle v_{\mu, k}^{*}, \tilde{\Phi}_{\mu, k}^{v}(z) v_{\lambda, k}\right\rangle=z^{-\Delta} v .
$$

Here $\hat{\otimes}$ denotes the completed tensor product.

Let $V_{1}, \ldots V_{r}$ be finite-dimensional $\mathfrak{g}$-modules, and let $v_{1} \in V_{1}, \ldots v_{r} \in V_{r}$ be homogeneous vectors of weight $\nu_{1}, \ldots \nu_{r}$ respectively. Set $\nu=\sum \nu_{i}$, and assume $\nu \in \mathfrak{l}^{\perp}$. We will consider composition of intertwining operators:

$$
\begin{aligned}
\tilde{\Phi}_{\mu-\nu_{2}-\ldots-\nu_{r}, k}^{v_{1}}\left(z_{1}\right) \ldots & \tilde{\Phi}_{\mu, k}^{v_{r}}\left(z_{r}\right) B e^{\lambda}: \\
& M_{\mu^{\prime}, k} \rightarrow M_{\mu-\nu, k} \otimes z_{1}^{-\Delta_{1}} V_{1}\left[z_{1}^{ \pm 1}\right] \otimes \ldots \otimes z_{r}^{-\Delta_{r}} V_{r}\left[z_{r}^{ \pm 1}\right],
\end{aligned}
$$

where $\lambda \in\left(\mathfrak{h}^{*}\right)^{B}, \mu \in \mathfrak{h}^{*}$, where $\left(\tilde{\mu}^{\prime}, \alpha\right)=(\tilde{\mu}, T \alpha)$ for $\alpha \in \Gamma_{1}$, and $\Delta_{i}=$ $g \frac{2\left(\nu_{i}, \mu-\nu_{i+1}-\ldots-\nu_{r}\right)-\left(\nu_{i}, \nu_{i}\right)}{2(k+g)}$. This composition lives in a certain completion (see [EFK1], §3) of the space

$$
\operatorname{Hom}\left(M_{\mu^{\prime}, k}, M_{\mu-\nu, k} \otimes V_{1} \otimes \ldots \otimes V_{r}\right) \otimes z_{1}^{-\Delta_{1}} \ldots z_{r}^{-\Delta_{r}} \mathbb{C}\left[z_{1}^{ \pm 1}, \ldots, z_{r}^{ \pm 1}\right] .
$$

Let us fix some $\tau \in \mathbb{C}$. For $\lambda \in \mathfrak{h}^{*}$ set $\tilde{\lambda}=\lambda+2 \pi i \tau \delta / g$. Consider the trace function

$$
F_{k}^{v_{1}, \ldots v_{r}}(\lambda, \mu, \mathbf{z})=\operatorname{Tr}\left(\tilde{\Phi}_{\mu-\nu_{2}-\ldots-\nu_{r}, k}^{v_{1}}\left(z_{1}\right) \ldots \tilde{\Phi}_{\mu, k}^{v_{r}}\left(z_{r}\right) B e^{\tilde{\lambda}}\right),
$$

where $\mathbf{z}=\left(z_{1}, \ldots, z_{r}\right)$. Finally, let

$$
\hat{\delta}_{B}(\lambda)=\left(\operatorname{Tr}_{\mid M_{0, k}}\left(B e^{\tilde{\lambda}}\right)\right)^{-1}
$$

and set

$$
\mathbb{F}_{k}^{v_{1}, \ldots v_{r}}(\lambda, \mu, \mathbf{z})=\hat{\delta}_{B}(\lambda) F_{k}(\lambda, \mu, \mathbf{z}) .
$$

Our main results in the affine case are

Theorem 5.1. The function $\mathbb{F}_{k}^{v_{1}, \ldots v_{r}}(\lambda, \mu, \mathbf{z})$ satisfies the following system of differential equations for $i=1, \ldots r$ :

$$
\begin{aligned}
& -\frac{k+g}{g} z_{i} \frac{\partial}{\partial z_{i}} \mathbb{F}_{k}^{v_{1}, \ldots v_{r}}(\lambda, \mu, \mathbf{z})=\sum_{j \in I_{1}} x_{j \mid V_{i}} \frac{\partial}{\partial x_{j}} \mathbb{F}_{k}^{v_{1}, \ldots v_{r}}(\lambda, \mu, \mathbf{z}) \\
& +\left(\sum_{j>i} \bar{r}_{T}\left(\lambda, z_{i} / z_{j}\right)_{\mid V_{i} \otimes V_{j}}-\sum_{j<i} \bar{r}_{T}\left(\lambda, z_{j} / z_{i}\right)_{\mid V_{j} \otimes V_{i}}\right) \mathbb{F}_{k}^{v_{1}, \ldots v_{r}}(\lambda, \mu, \mathbf{z}) .
\end{aligned}
$$

These equations are called the KZB equations (see $[\mathrm{F}]$ ). 
Theorem 5.2. The function $\mathbb{F}^{v_{1}, \ldots, v_{r}}(\lambda, \mu, \mathbf{z})$ satisfies the following second order differential equation:

$$
\begin{aligned}
-\frac{k+g}{\pi i} \frac{\partial}{\partial \tau} \mathbb{F}_{k}^{v_{1}, \ldots v_{r}}(\lambda, \mu, \mathbf{z})= & \\
& \sum_{j \in I_{1}} \frac{\partial^{2}}{\partial x_{j}^{2}} \mathbb{F}_{k}^{v_{1}, \ldots v_{r}}(\lambda, \mu, \mathbf{z})-\sum_{i, j=1}^{r} \bar{S}^{i j}\left(\lambda, z_{i} / z_{j}\right) \mathbb{F}_{k}^{v_{1}, \ldots v_{r}}(\lambda, \mu, \mathbf{z})
\end{aligned}
$$

where

$$
\bar{S}_{T}(\lambda, z):=\left(e v_{z} \otimes e v_{1}\right)\left(\phi^{-1} \otimes \phi^{-1}\right)\left(S_{T}(\lambda)\right)
$$

and $S_{T}(\lambda)$ is the function defined in Theorem 3.2 (for $\tilde{\mathfrak{g}}$ ).

The proofs of these theorems are completely parallel to the proofs of the theorems of Section 3.

Remark 1. If $\Gamma_{1}=\Gamma_{2}=\Gamma$ then $\bar{S}_{T}(\lambda, z)$ can be expressed in terms of elliptic functions. In general, this function expresses via trigonometric and elliptic functions.

Remark 2. In section 3 we saw that the differential operator $D=\Delta_{\mathfrak{l}}-S_{T}(\lambda)$ (where $\Delta_{\mathfrak{l}}$ is the Laplacian of $\mathfrak{l}$ ) on functions with values in the space of weight zero under $\mathfrak{l}$ in some representation of the Lie algebra can be included in a quantum integrable system. A similar statement holds for the operator $D_{\tau}=$ $\Delta_{\mathfrak{l}}-\bar{S}_{T}(\lambda, 1)$, whose coefficients are elliptic (if $\Gamma_{i}=\Gamma$ ). To obtain other operators commuting with $D_{\tau}$, it is necessary to apply the construction of Section 3.5 to central elements of a completion of $U(\tilde{\mathfrak{g}})$ at the critical level $k=-g$, as explained in [EFK2]. In the case $T=1$, this gives a generalization of the elliptic CalogeroMoser system (see [EFK2]).

Remark 3. Although we considered only untwisted affine algebras, the results can be easily generalized to the twisted case.

Acknowledgments. P.E. would like to thank IHES for hospitality. O.S. is grateful to the Harvard Mathematics Dept. for the kind invitation. The work of P.E was partially supported by the NSF grant 9700477 , and was partly done when P.E. was employed by the Clay Mathematical Institute as a CMI prize fellow. P.E. is grateful to Travis Schedler for useful discussions.

\section{References}

[BD] A. Belavin and V. Drinfeld, Triangle equations for simple Lie algebras, Math. Phys. Rev., 4 (ed. Novikov et al.), pp. 93-165, Harwood Academic Publ., New York, 1984.

[B] D. Bernard, On the Wess-Zumino-Witten model on the torus, Nucl. Phys B 303 (1988), 77-93.

[E] P. Etingof, Representations of affine Lie algebras, elliptic r-matrix systems and special functions, Comm. Math. Phys. 159 (1994) 471-502.

[EFK1] P. Etingof, I. Frenkel, A. Kirillov, Jr., Lectures on representation theory and KnizhnikZamolodchikov equations, Mathematical Surveys and Monographs, 58, American Mathematical Society, Providence, RI, 1998.

[EFK2] _ Spherical functions on affine Lie groups, Duke Math. J. 80 (1995), 59-90. 
[EV1] P. Etingof and A. Varchenko, Geometry and classification of solutions of the classical dynamical Yang-Baxter equation, Comm. Math. Phys. 192 (1998), 77-120.

[EV2] Exchange dynamical quantum groups, preprint, math.QA/9801135, to appear in Comm. Math. Phys.

[EV3] Traces of intertwiners for quantum groups and difference equations, I, math.QA 9907181, submitted to Duke Math. J.

[F] G. Felder, Conformal field theory and integrable systems associated to elliptic curves, Proceedings of the International Congress of Mathematicians, Vol. 1, 2 (Zürich, 1994), pp. 1247-1255, Birkhäuser, Basel, 1995.

[K] V. Kac, Infinite dimensional Lie algebras, Cambridge University Pres, Cambridge, (1990).

[KK] V.G. Kac and D. Kazhdan, Structure of representations with highest weight of infinitedimensional Lie algebras, Adv. in Math. 34 (1979), 97-108.

[KT] G. Kuroki and T. Takebe, Twisted Wess-Zumino-Witten models on elliptic curves, Comm. Math. Phys. 190 (1997) 1-56.

[S] O. Schiffmann, On classification of dynamical r-matrices, Math. Res. Lett., 5 (1998), 13-30.

Department of Mathematics, 2-165, Mit, 77 Mass. Ave, Cambridge, MA 02139

E-mail address: etingof@math.mit.edu

Department of Mathematics, Mit, 77 Mass. Ave, Cambridge, MA 02139

E-mail address: schiffma@clipper.ens.fr 